\title{
Extraordinary accuracy in floret position of Helianthus annuus
}

\author{
Takuya Okabe* \\ Graduate School of Integrated Science and Technology, Shizuoka University, 3-5-1 Johoku, Naka-ku, 432-8561 Hamamatsu, Japan
}

\begin{abstract}
Divergence angles were measured for inflorescences of Helianthus annuus with several hundreds to more than a thousand disk flowers. Quantitative analysis showed that the angles are robustly fixed in the vicinity of the ideal golden angle $137.508^{\circ}$ as accurately as $\sim 0.001^{\circ}$. The mean deviation from the ideal value varies for each sample. The results have important implications for phyllotaxis models, which are discussed by referring to a necessary modification proposed by Roberts.
\end{abstract}

Keywords: phyllotaxy; Asteraceae; contact pressure model; expanding apex model; Hofmeister’s rule

\section{Introduction}

Phyllotaxis patterns of leaves or modified leaves in general on a plant stem are classified into several types and each type as well as its transformation are rich in diversity. Distichy and simple spiral patterns have one leaf at each node, while decussate and whorled patterns have multiple leaves at a node. Each spiral direction occurs with equal probability [1]. Some plants may have interestingly variable patterns, e.g., a female catkin of Betula alba, an ear of Plantago major, bracts of $P l$. media [1], a vegetative shoot of Abies balsamea [2], gynoecium of Magnolia acuminata [3,4], capitulum of Carlina acaulis [5], primary vascular system of Anagallis arvensis [6], areoles of the family Cactaceae [7]. Thus there is no doubt that not all phyllotaxis properties are genetically determined. Independently of the diversity problems, simple spiral phyllotaxis in itself is especially noted not only for its predominant occurrence but because divergence angles between successive leaves are precisely fixed at a very special value. The spiral arrangement is so precise that Hirmer particularly called it "spiral phyllotaxis with precise divergences" to distinguish from variable counterparts related to distichy and decussate systems [8]. It is interesting to note that, in the family Cactaceae, the tribe Cacteae in which the simple spiral pattern dominates has more precise divergence angles than other tribes in which the decussate pattern occurs with high frequency [7].

Normally, the divergence angle of a simple spiral pattern is intriguingly close to the golden angle $\alpha_{\text {gold }}=360 /(1+\tau) \cong$ $137.508^{\circ}$, which is the smaller one of the two angles created by sectioning the circumference of a full circle according to the golden proportion: $1: \tau=\tau-1: 1 \cong 1: 1.618$.

\footnotetext{
*Email: okabe.takuya@shizuoka.ac.jp
}

Handling Editor: Beata Zagórska-Marek
The invariability of divergence angle was already remarked by Bravais and Bravais [9] (p. 69, 106): "By direct measurements, we find constantly an angle differing very little from $137^{\circ}$ or $138^{\circ}$, and this precision may be very surprising if we note that on a stem of average size, for example a centimeter in diameter, the arc of $1^{\circ}$ does not exceed the 8 hundredth of a millimeter, a quantity almost inappreciable to the naked eye.." "The genetic spiral extends to the underground stem by keeping the same invariable divergence, and even to the organs of the flower.". The accurate occurrence of the limit divergence $\alpha_{\text {gold }}$ was underscored by van Iterson [10]. Comparing with his theory, he wrote (p. 247): "However, especially in cases of the contact 1 and 2 at the apex, the trouble becomes apparent. While the theory allows all divergences between $180^{\circ}$ and $128.5^{\circ}$ as possible for this contact, one finds only divergences between $137^{\circ}$ and $142^{\circ}$, namely a great approach to the limit divergence of the main sequence.. The uniqueness of the golden angle was strongly supported by Hirmer and his colleagues (see references cited in [11]). To cite an example, for composite flowers of Rudbeckia laciniata, Lepachys pinnatifida, Chrysanthemum millefoliatum, Bidens leucantha, Galinsoga parvifiora, Cineraria lobata and others, it was argued that the number of ray florets tends to be Fibonacci numbers like 5, 8, 13, 21, 34 because the ray florets are regularly arranged with the golden angle [8]. The universality of the golden angle was directly demonstrated by Fujita [11], who measured divergence angles in bud sections of thirty species of flowering plants with one degree accuracy. Showing distribution curves conspicuously peaked at $137^{\circ}$ or $138^{\circ}$, he remarked: "From these data we can see that the peak point of the variation curve of divergence comes strongly close to the limit value, though with a large variation width. In other words, in the regular spiral arrangement, the divergence follows the limit value, as Hirmer claims, independently of the numbers of conjugate parastichies.". Snow suggested that the golden 
angle might have some biological advantage by referring to the difficulty of van Iterson underlined by this result of Fujita [12]. As for sample variance, it was reported for young shoots of Erigeron sumatrensis that each shoot has an individual deviation from the golden angle and the mean average was $137.499^{\circ} \pm 0.212^{\circ}$ [13]. The first quantitative analysis of floret patterns of Helianthus was made by Ryan et al. [14]. They reported systematic and persistent fluctuations of $\pm 5^{\circ}$ about the ideal value and concluded that capitula do not possess a uniquely defined growth center. As reported for Helianthus annuus [15], divergence angles of mature leaves are not measured with a better accuracy because of secondary twist of the stem. As a matter of fact, secondary deformation of the phyllotaxis pattern generally causes apparent fluctuations in divergence angle [16]. Therefore, measured angles may depend on the procedure to determine the center position of the phyllotaxis pattern [17]. Accordingly, a true deviation from the ideal value $\alpha_{\text {gold }}$ is almost always bound to be hidden beneath various practical errors by chance. Nevertheless, by whatever mechanism it occurs, there is no doubt that any particular real life system has a more or less definite deviation from the mathematical ideality $\alpha_{\text {gold }}=137.50776 \ldots$. The present study makes a first attempt at detecting and assessing a statistically significant deviation from the ideality for a representative system of spiral phyllotaxis. To suppress statistical errors in a systematic manner, a large number of divergence angles have to be collected from an individual sample. Inflorescences of the Asteraceae are the best suited to this end. In fact, as it turns out, they are perhaps the one and only subject with which to achieve the purpose. This paper reports that accuracy in divergence angle of Helianthus annuus amounts to as high as $\sim 0.001^{\circ}$. Although this may seem implausible at first, it is immediately obvious by observing how regularly Fibonacci numbers (1, 2, 3, 5, 8, $13,21,34,55,89,144,233,377,610,987$, etc.) are arranged in Fig. 1 and Fig. 2. The measurement results of this study reinforce evidence for the accurate constancy of divergence angle to an unquestionable degree and may shed new light on the fundamental problem of phyllotaxis.

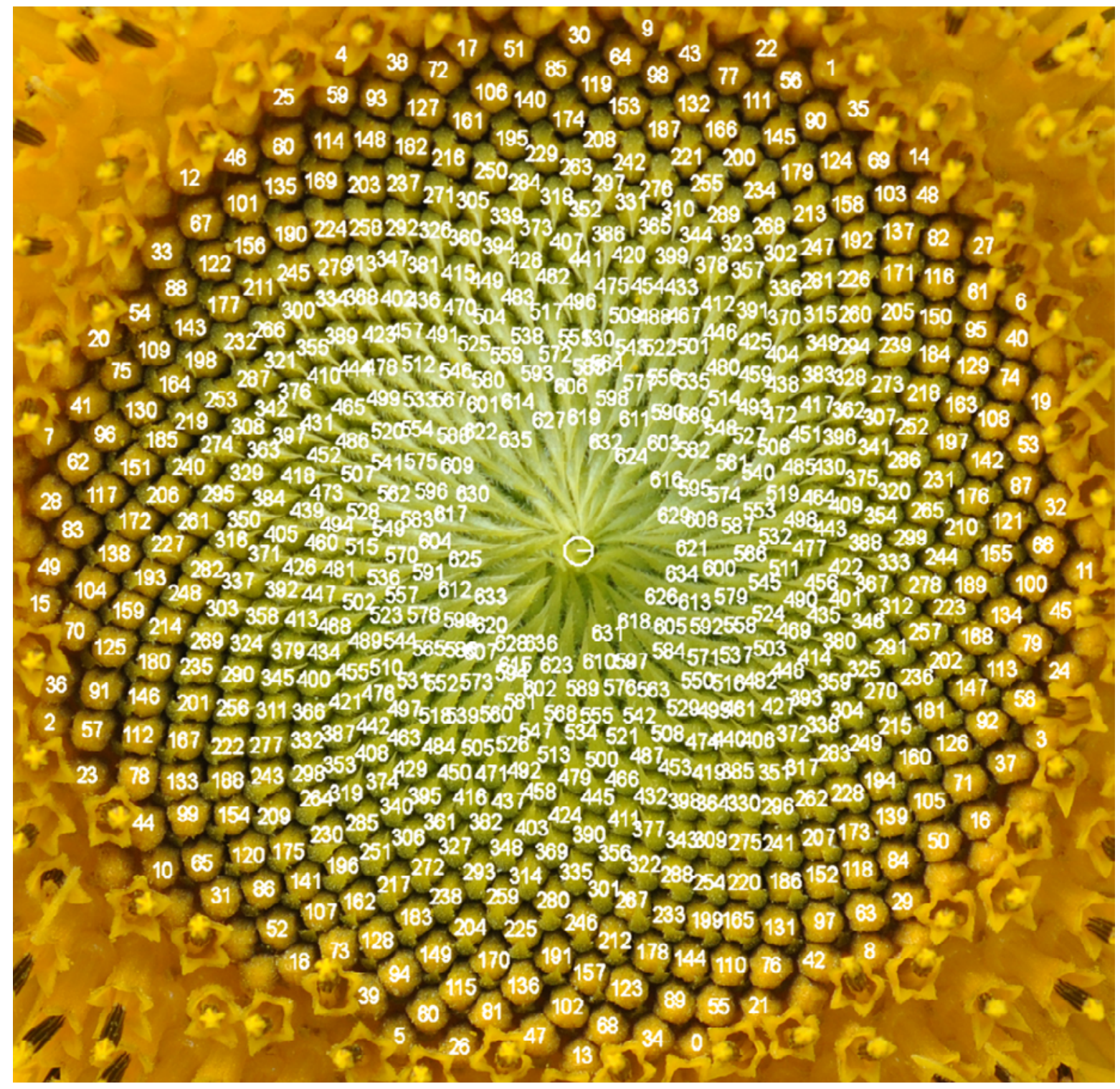

Fig. 1 A sunflower head with divergence angle $\alpha \simeq 137.513 \pm 0.003^{\circ}$. Disk florets are numbered algorithmically [16]. They are formed counterclockwise inward. Adapted from Yellow sunflower 001/Wikimedia Commons. 


\section{Material and methods}

The divergence angle $\alpha$ is evaluated by measuring the angle $\angle P_{n} O P_{n+q}$ subtended by two arbitrary florets $P_{n}$ and $P_{n+q}$, where $O$ denotes a fixed center [9]. Solving

$$
\angle P_{n} O P_{n+q}=q \alpha-360 p
$$

for $\alpha$,

$$
\alpha_{n}(q)=\left(\angle P_{n} O P_{n+q}+360 p\right) / q .
$$

This is the main formula used in this study. The angle $\angle P_{n} O P_{n+q}$ is measured in the direction of the genetic spiral, that is, the imaginary spiral connecting all the florets in increasing order of the index $n$, so that the divergence angle $\alpha_{n}(q)$ is a positive number. On the right-hand side of Equation (1), an integer multiple of full turns, $360 p$ in degrees of arc, is subtracted to reduce the total angle $q \alpha\left(>360^{\circ}\right)$ to a net angle within $\pm 180^{\circ}$. The angle $\angle P_{n} O P_{n+q}$ is calculated from the $x y$-coordinates of two vectors $O P_{n}$ and $O P_{n+q}$. The coordinates of the floret position $P_{n}$ are digitally read from photographic images published in media file repositories, which are now of ideal quality incomparable with any figures in published papers. For indexing and data collection, the algorithm described previously was applied [16]. As the angle $\angle P_{n} O P_{n+q}$ comprises $q$ divergence angles, the effect of secondary disturbances on each divergence angle is averaged out when the integer $q$ is large enough. As a rough guide, the standard error in measuring $\angle P_{n} O P_{n+q}$ is typically less than 1 degree. Accordingly, accuracy of $\alpha_{n}(q)$ is less than about $1 / q$ degree. As $\alpha_{n}(q)$ turns out to be very close to the golden angle, it is not necessary but convenient to employ Fibonacci numbers for $q$ and $p$ in the above formula, namely $(q, p)=$ $(1,0),(2,1),(3,1),(5,2),(8,3),(13,5)$ and so on.

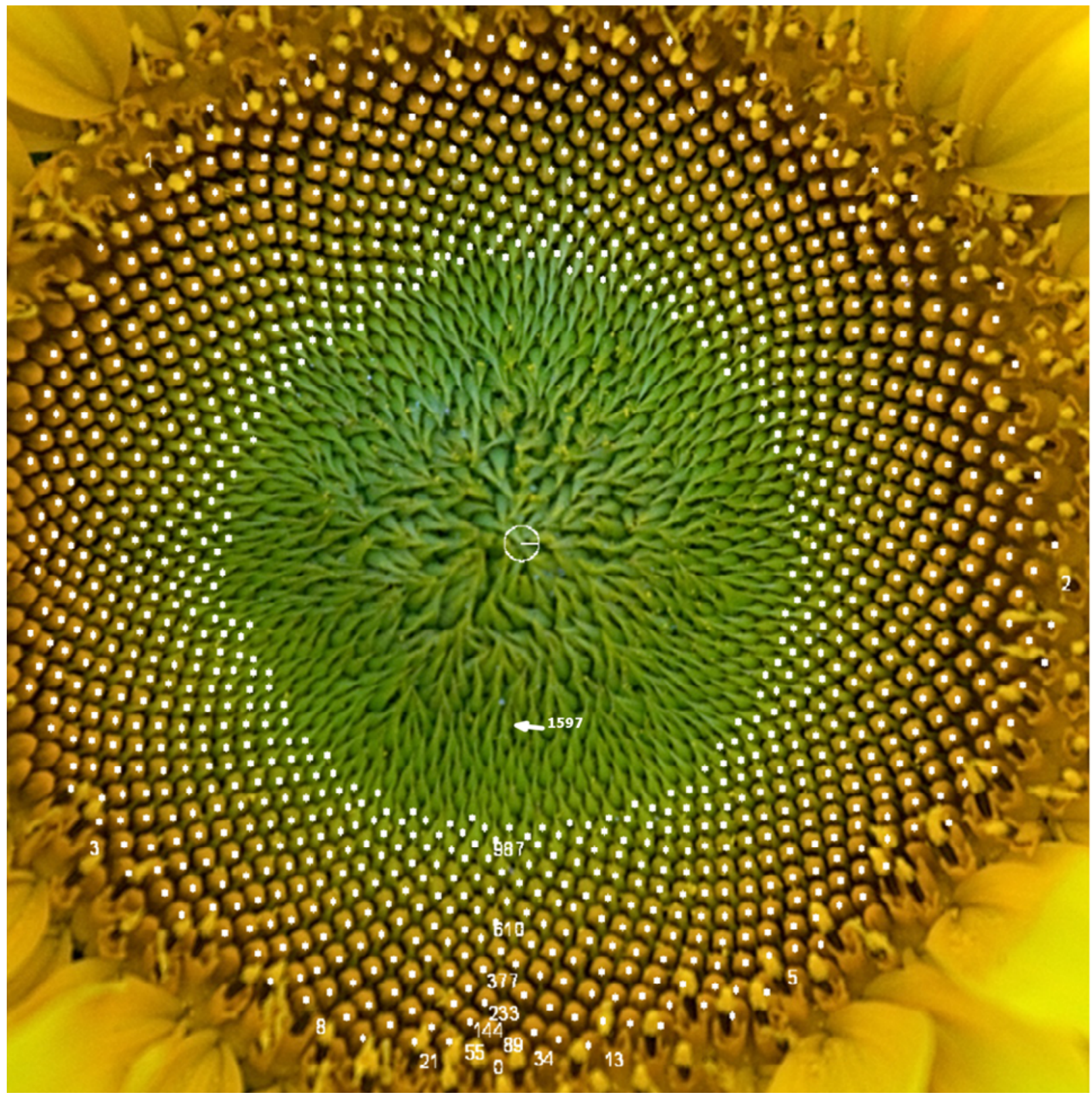

Fig. 2 A large sunflower head with $\alpha \simeq 137.507 \pm 0.001^{\circ}$. The phyllotaxis fraction is as high as $377 / 987$. Florets are formed clockwise inward. Adapted from Sunflower Closeup Hungary/Wikimedia Commons. 


\section{Results}

The divergence angle $\alpha_{n}(q)$ was evaluated for disk florets of Fig. 1 . The $n$-dependence of $\alpha_{n}(q)$ is qualitatively similar for any value of $q$, whereas the variation range depends on $q$. In Fig. 3, $\alpha_{n}(233)$ is plotted against the floret index $n$ representatively. The mean and standard deviation of $\alpha_{n}(233)$ are 137.5139 $\pm 0.0041,137.5145 \pm 0.0038,137.5080 \pm 0.0060$, $137.5000 \pm 0.0155$ for $n \leq 100,100<n \leq 200,200<n \leq 300$, $300<n$, respectively. Thus, $\alpha_{n}(q)$ is robustly constant over a wide range of $n$. This is surprising and not at all self-evident because the hundreds of florets were formed under varying conditions. The mean variation is about the same order as statistical errors, namely $0.01^{\circ}$ at most. To suppress statistical noise including measurement error in $\angle P_{n} O P_{n+q}$, florets near the center were excluded from the statistical analysis. Tab. 1 presents the mean $\langle\alpha\rangle$ and standard deviation $\sigma$ of $\alpha_{n}(q)$ evaluated by using the first 400 florets of Fig. 1. The constant mean indicates that the florets are formed in accordance with a fixed divergence angle $\simeq 137.51^{\circ}$. On the other side, the standard deviation $\sigma$ decreases as $q$ increases, as expected. If the deviation $\sigma$ is interpreted as a result of $q$ independent random variations of individual divergence angles $\delta \alpha$ [cf. Equation (1)], then

$$
\sigma \approx \delta \alpha / \sqrt{q}
$$

This is a standard result of statistical theory. The results for large $q$ in Tab. 1 are consistent with this relation for $\delta \alpha \simeq 0.06^{\circ}$, which is also remarkable. The deviation $\sigma$ for a small $q$ represents the persistent fluctuations mentioned in Introduction, which therefore does not follow this relation. Owing to $\sigma$ being suppressed systematically, the mean value begins to show a clear sign of deviation from the ideal value, that is, $\langle\alpha\rangle-\alpha_{\text {gold }}>1.5 \sigma$. See Fig. 3 and Tab. 1. This is supported by consistent results in Tab. 2 and Tab. 3 for other independent samples. Tab. 4 presents the results for a larger sample of Fig. 2. Tab. 5 is for a packing pattern of mature seeds. The seed pattern has an order of magnitude larger values of $\sigma$ and $\delta \alpha$, while the deviation $\langle\alpha\rangle-\alpha_{\text {gold }}$ is not affected comparatively. Thus it appears that the floret patterns are not altered substantially by the growth that follows their establishment.

These results indicate that the deviation $\langle\alpha\rangle-\alpha_{\text {gold }}$ is robust and typically of the order of a $10^{-3}$ degree. To suppress $\sigma$ to this order, the sample size, or $q$ for Equation (3), has to be larger than $\sim 100$ even when $\delta \alpha$ is as small as $0.05^{\circ}$. Thus, in hindsight, the necessary condition for detecting the deviation is barely satisfied for outer florets of the sunflower capitula. It is not asserted from the present study alone whether the higher accuracy of $\langle\alpha\rangle-\alpha_{\text {gold }} \simeq 10^{-4}$ in Tab. 4 is due to a large capitulum size or just a coincidence. It is interesting to see whether the minute individual difference identified in this study is used as a fingerprint of each plant. In fact, the flower head of Fig. 1 is distinguished from that of Fig. 2 by their different divergence angles, though the difference of $0.007^{\circ}$ is too small to be noticed without special attention.

\section{Discussion}

Schwendener, Church and van Iterson attempted to explain the tendency to the limit divergence $\alpha_{\text {gold }}$ by postulating that contact parastichies tend to cross at right angles (p. 248 [10]; p. 340 [18]; see also [19]). They were criticized because the predicted angles are too close to $\alpha_{\text {gold }}$ to test empirically [8] and when the predicted deviation is large enough it is disproved by observation [20]. As a matter of fact, the crossing angles of contact parastichies are not even constant. The angle $\angle P_{n+34} P_{n} P_{n+55}$ in Fig. 1 varies from $116^{\circ}$ for $n=0$ to $49^{\circ}$ for $n=444$. In fact, divergence angles remain constant independently of the crossing angles of parastichies.

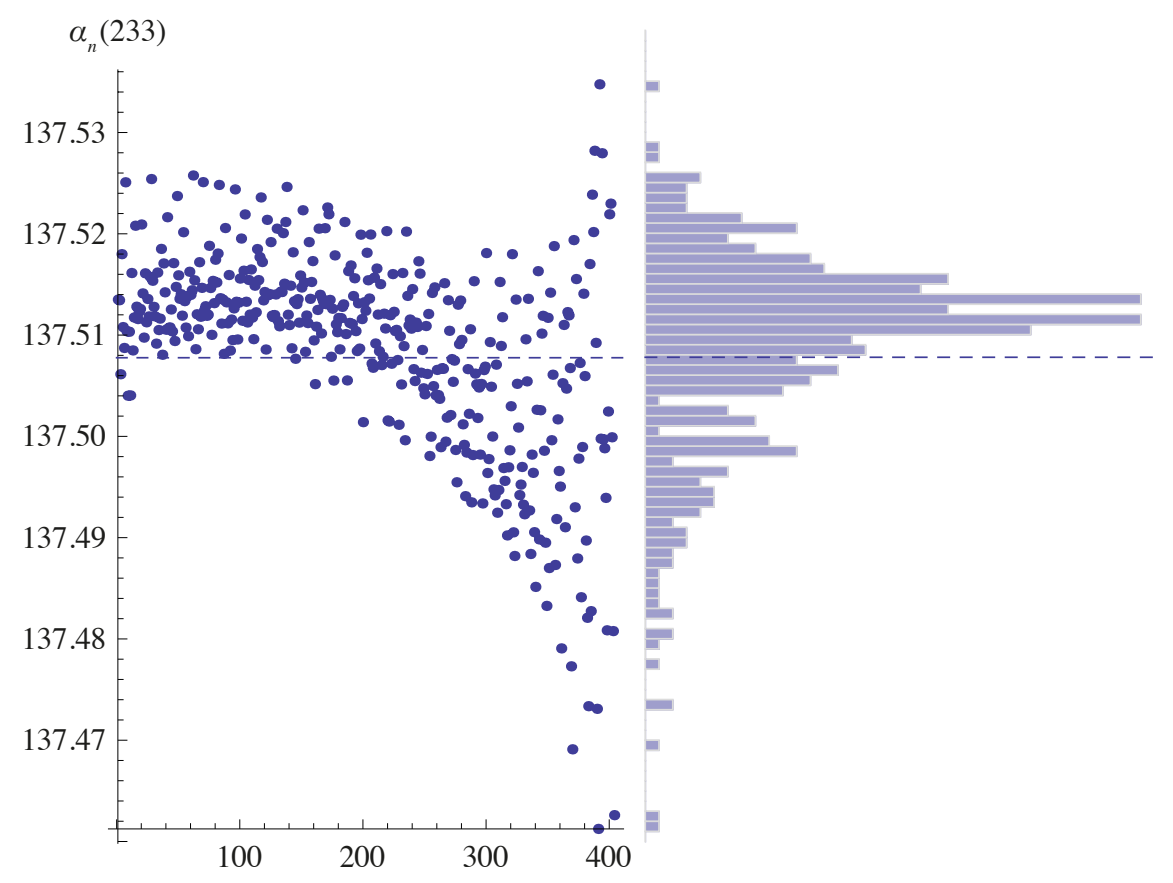

Fig. 3 The divergence angle $\alpha_{n}(233)$ for the pattern of Fig. 1 is plotted against the floret index $n$. The ideal golden angle of $137.508^{\circ}$ is indicated by a dashed line. 
Tab. 1 Divergence angles of disk flowers of a sunflower.

\begin{tabular}{rccccc}
$\boldsymbol{q}$ & $\langle\boldsymbol{\alpha}\rangle$ & $\boldsymbol{\sigma}$ & $\sqrt{\boldsymbol{q} \sigma}$ & $\langle\boldsymbol{\alpha}\rangle-\boldsymbol{\alpha}_{\text {gold }}$ & $\left(\langle\boldsymbol{\alpha}\rangle-\boldsymbol{\alpha}_{\text {gold }}\right) / \boldsymbol{\sigma}$ \\
\hline 1 & 137.5054 & 2.2337 & 2.234 & -0.0024 & \\
2 & 137.5115 & 1.1829 & 1.673 & 0.0038 & 0.003 \\
3 & 137.5130 & 0.6575 & 1.139 & 0.0052 & 0.08 \\
5 & 137.5132 & 0.2970 & 0.664 & 0.0055 & 0.02 \\
8 & 137.5126 & 0.1337 & 0.378 & 0.0048 & 0.04 \\
13 & 137.5121 & 0.0605 & 0.218 & 0.0043 & 0.07 \\
21 & 137.5130 & 0.0294 & 0.135 & 0.0053 & 0.18 \\
34 & 137.5135 & 0.0147 & 0.086 & 0.0057 & 0.39 \\
55 & 137.5136 & 0.0089 & 0.066 & 0.0059 & 0.66 \\
89 & 137.5139 & 0.0064 & 0.061 & 0.0062 & 0.96 \\
144 & 137.5142 & 0.0047 & 0.056 & 0.0064 & 1.4 \\
233 & 137.5142 & 0.0040 & 0.061 & 0.0064 & 1.6 \\
\hline
\end{tabular}

The mean $\langle\alpha\rangle$ and standard deviation $\sigma$ of the divergence angle $\alpha_{n}(q)$ are evaluated for first 400 disk flowers of Fig. 1 . The ideal golden angle is given by $\alpha_{\text {gold }}=137.50776^{\circ}$. The first row for $q=1$ corresponds to the standard method of evaluating the angle between successive florets.

Tab. 2 Divergence angles of disk flowers of a sunflower.

\begin{tabular}{rccccc}
\hline $\boldsymbol{q}$ & $\langle\boldsymbol{\alpha}\rangle$ & $\boldsymbol{\sigma}$ & $\sqrt{\boldsymbol{q}} \boldsymbol{\sigma}$ & $\langle\boldsymbol{\alpha}\rangle-\boldsymbol{\alpha}_{\text {gold }}$ & $\left(\langle\boldsymbol{\alpha}\rangle-\boldsymbol{\alpha}_{\text {gold }}\right) / \boldsymbol{\sigma}$ \\
\hline 1 & 137.5143 & 2.9275 & 2.928 & 0.0065 & \\
34 & 137.5113 & 0.0182 & 0.106 & 0.0035 & 0.19 \\
55 & 137.5117 & 0.0113 & 0.084 & 0.0039 & 0.34 \\
89 & 137.5121 & 0.0078 & 0.073 & 0.0043 & 0.56 \\
144 & 137.5125 & 0.0053 & 0.064 & 0.0048 & 0.90 \\
233 & 137.5126 & 0.0042 & 0.064 & 0.0048 & 1.1 \\
\hline
\end{tabular}

The mean $\langle\alpha\rangle$ and standard deviation $\sigma$ of the divergence angle $\alpha_{n}(q)$ for 400 disk flowers of a sunflower head with about 580 florets and a parastichy pair of $(34,55)$.

Tab. 3 Divergence angles of disk flowers of a sunflower.

\begin{tabular}{rccccc}
\hline $\boldsymbol{q}$ & $\langle\boldsymbol{\alpha}\rangle$ & $\boldsymbol{\sigma}$ & $\sqrt{\boldsymbol{q}} \boldsymbol{\langle \alpha}\rangle-\boldsymbol{\alpha}_{\text {gold }}$ & $\left(\langle\boldsymbol{\alpha}\rangle-\boldsymbol{\alpha}_{\text {gold }}\right) / \boldsymbol{\sigma}$ \\
\hline 1 & 137.4909 & 2.7422 & 2.742 & -0.0169 & \\
34 & 137.4988 & 0.0251 & 0.147 & -0.0089 & -0.36 \\
55 & 137.4984 & 0.0171 & 0.127 & -0.0094 & -0.55 \\
89 & 137.4986 & 0.0134 & 0.127 & -0.0092 & -0.68 \\
144 & 137.4988 & 0.0096 & 0.115 & -0.0090 & -0.93 \\
233 & 137.4987 & 0.0047 & 0.072 & -0.0091 & -1.9 \\
\hline
\end{tabular}

The mean $\langle\alpha\rangle$ and standard deviation $\sigma$ of the divergence angle $\alpha_{n}(q)$ for 330 disk flowers of a sunflower head with about 380 florets and a parastichy pair of $(34,55)$.
Tab. 4 Divergence angles of disk flowers of a sunflower.

\begin{tabular}{rccccc}
\hline $\boldsymbol{q}$ & $\langle\boldsymbol{\alpha}\rangle$ & $\boldsymbol{\sigma}$ & $\sqrt{\boldsymbol{q}} \boldsymbol{\langle \alpha}\rangle-\boldsymbol{\alpha}_{\text {gold }}$ & $\left(\langle\boldsymbol{\alpha}\rangle-\boldsymbol{\alpha}_{\text {godd }}\right) / \boldsymbol{\sigma}$ \\
\hline 1 & 137.5067 & 2.2778 & 2.278 & -0.0010 & \\
55 & 137.5072 & 0.0067 & 0.050 & -0.0006 & -0.09 \\
89 & 137.5072 & 0.0043 & 0.041 & -0.0006 & -0.14 \\
144 & 137.5072 & 0.0028 & 0.034 & -0.0006 & -0.21 \\
233 & 137.5071 & 0.0022 & 0.034 & -0.0006 & -0.29 \\
377 & 137.5070 & 0.0016 & 0.032 & -0.0008 & -0.46 \\
610 & 137.5069 & 0.0013 & 0.031 & -0.0009 & -0.67 \\
987 & 137.5071 & 0.0010 & 0.031 & -0.0006 & -0.64 \\
\hline
\end{tabular}

The mean $\langle\alpha\rangle$ and standard deviation $\sigma$ of the divergence angle $\alpha_{n}(q)$ for 1161 florets among the total number of about 1700 in Fig. 2.

Tab. 5 Divergence angles of sunflower seeds.

\begin{tabular}{rccccc}
$\boldsymbol{q}$ & \multicolumn{1}{c}{$\langle\boldsymbol{\alpha}\rangle$} & \multicolumn{1}{c}{$\boldsymbol{\sigma}$} & $\sqrt{\boldsymbol{q} \sigma}$ & $\langle\boldsymbol{\alpha}\rangle-\boldsymbol{\alpha}_{\text {gold }}$ & $\left(\langle\boldsymbol{\alpha}\rangle-\boldsymbol{\alpha}_{\text {gold }}\right) / \boldsymbol{\sigma}$ \\
\hline 1 & 137.5117 & 5.077 & 5.077 & 0.0039 & \\
34 & 137.5112 & 0.0256 & 0.149 & 0.0034 & 0.13 \\
55 & 137.5112 & 0.0204 & 0.151 & 0.0034 & 0.17 \\
89 & 137.5119 & 0.0175 & 0.165 & 0.0041 & 0.24 \\
144 & 137.5125 & 0.0163 & 0.196 & 0.0047 & 0.29 \\
233 & 137.5126 & 0.0153 & 0.234 & 0.0049 & 0.32 \\
\hline
\end{tabular}

For a seed packing. The mean $\langle\alpha\rangle$ and standard deviation $\sigma$ of the divergence angle $\alpha_{n}(q)$ for 400 seeds of a sunflower head with about 550 florets and a parastichy pair of $(34,55)$.

Roberts [21] proposed to make up deficiencies of phyllotaxis models [22,23]. One of them concerns the accuracy problem. His remark applies to any model based on the premise that the position of a new primordium is determined by the existing primordia. The difficulty has been left unaddressed in recent models $[24,25]$. The pattern in which $P_{n}$ has contact with $P_{n-q}$ and $P_{n-q}$ is called a $\left(q, q^{\prime}\right)$ system. In any pattern, including a $(1,2)$ system, a correct model should put $P_{3}$ at the golden section point in the largest gap between $P_{1}$ and $P_{2}$ (Appendix S1). To account for this, Roberts proposed to modify the premise such that the position of a new primordium (e.g., $P_{3}$ ) is determined not by its near neighbors (e.g., $P_{1}$ and $P_{2}$ ) but by older primordia remotely located from it (e.g., $P_{0}$ and $P_{-2}$ ). In a word, it is equivalent to postulating that internal or "chemical" contact of the organs is of higher order than it appears from the outside. To meet the accuracy observed in this study, the chemical contact has to be supposed as high as $(89,144)$, even if the pattern appears to be $(34,55)$. Take Fig. 1 for example. The mean and standard deviation of $\angle P_{n-89} O P_{n}$ and $\angle P_{n-144} O P_{n}$ are $-1.3 \pm 0.6<0$ and $2.0 \pm 0.7>0$, respectively. The fact that the former (latter) is negative (positive) means that $P_{n}$ lies on the negative (positive) side of $O P_{n-89}\left(O P_{n-144}\right)$. Accordingly, 
$P_{n}$ lies between the narrow angle $\angle P_{n-144} O P_{n-89} \cong 3^{\circ}$ (in the gap $\angle P_{n-55} O P_{n-34} \cong 8^{\circ}$ ). See Fig. 1. $P_{377}$ lies between $P_{233}$ and $P_{288}$. For the sake of argument, let it be assumed that $P_{n}$ is determined by $P_{n-89}$ and $P_{n-144}$ (i.e., $P_{377}$ by $P_{288}$ and $\left.P_{233}\right)$. Then their interaction, of whatever sort, has to be such as to meet the following requirements. (i) Constancy problem. For each sample, the angle $\angle P_{n-q} O P_{n}$ is fixed independently of the floret $n$. (ii) Individuality problem. The fixed angle $\angle P_{n-q} O P_{n}$ varies for each sample. The former refers to the robustness of $\alpha_{n}(q)-$ cf. Fig. 3. This is evidenced by $\angle P_{n-34} O P_{n}=-4.5 \pm 0.5$, $\angle P_{n-55} O P_{n}=3.2 \pm 0.5$ and $\angle P_{n-89} O P_{n}=-1.3 \pm 0.6$ for Fig. 1 . The latter refers to the sample dependence of $\alpha_{n}(q)$. Remark that $\alpha_{n}(q)$ by Equation (2) is given by the angle $\angle P_{n} O P_{n+q}$, which is determined by the assumed interaction. To illustrate (ii), $\angle P_{n-89} O P_{n}=-1.3 \pm 0.6$ for Tab. 1 is contrasted with $\angle P_{n-89} O P_{n}$ $=-2.8 \pm 1.2$ for Tab. 3. For reference, the pattern of Fig. 2 has $\angle P_{n-89} O P_{n}=-1.86 \pm 0.38$, whereas the exact golden angle $\alpha_{\text {gold }}$ gives $\angle P_{n-89} O P_{n}=-1.8090$. Furthermore, (iii) the angles satisfy the following relation:

$$
q \angle P_{n-q^{\prime}} O P_{n}-q^{\prime} \angle P_{n-q} O P_{n}= \pm 360^{\circ}
$$

where, for the normal phyllotaxis, $q$ and $q$ ' are successive Fibonacci numbers. The above $(i)$ signifies that $\alpha_{n}(q)$ is independent of $n$. This relation signifies that $\alpha_{n}(q)$ is independent of $q$, which is obvious from Tab. 1-Tab. 5. Indeed, for the left-hand side of the last equation, the pattern of Fig. 1 gives $360.0 \pm 6.2$ for $\left(q, q^{\prime}\right)=(2,3), 359.8 \pm 28.8$ for $(34,55)$, $355.6 \pm 62.8$ for $(89,144)$ and so on. To sum up, the observed results indicate that all angles $\angle P_{n-q} O P_{n}$ are determined by a single constant $\alpha_{n}(q)=\alpha$, which is minutely specific to an individual sample. Thus, Roberts' proposal creates more difficult problems.

The riddle of phyllotaxis is why the constant $\alpha$ is $\alpha_{\text {gold }}=$ $137.508^{\circ}$. The theoretical difficulty stems from the fundamental premise that divergence angles are determined by the existing primordia, or that the phyllotaxis system is "a dynamical system" (in the technical sense of mathematical physics) in that the angle $\angle P_{n} O P_{n-1}$ is determined by the preceding $P_{m}$ 's (i.e., $m<n$ ). This has been an unchallenged, working hypothesis since early times. There is a contrasting approach of explaining special traits of living things, according to which the robustness of divergence angles may be explained without understanding the molecular mechanism of developmental processes of primordia formation. It can be simply that plants have evolved so as to generate lateral organs at fixed intervals of angle and so the divergence angle is pre-determined independently of the existing primordia. Hence, it would be instructive to revisit the original motivation of the phyllotaxis models, which is stated in the opening paragraphs of van Iterson [10].

"The times are over when we content ourselves with describing and classifying manifestations of the plant world with great accuracy. Since the theory of evolution has taught us to consider a number of peculiarities of inner and outer constructions as functional for the plants, we have made efforts to explain morphological facts as manifestations of adaptation more and more. Although we are taken certainly too far in this endeavor, it cannot be denied that this way of treatment has produced the finest results and stimulated many new studies. In addition to this line of research, now a second has developed, in which, setting aside the question of functionality, one seeks to bring various manifestations into causal relationship, and whereby one seeks to interpret a property as the mechanical necessary consequence of certain others. Thereby the first way of treatment loses none of its importance, as natural selection will retain only a preservable one from different forms which are possible for mechanical reasons. Therefore those special cases in that functionality is least pronounced and different possibilities make their appearance in most complete forms will be most suitably carried through with the mechanical way of understanding.

Now, there are few morphological facts showing such regularity and peculiarity as the phenomena of phyllotaxis, as they became well-known to us particularly by the works of Schimper and Braun, A. and L. Bravais, and just right here the functionality is not at all obvious ${ }^{1)}$. So it is understandable that the most thorough attempts of carrying out a mechanical explanation have been made in this field. Here is pointed out only phyllotaxis theories of Hofmeister, Airy, Delpino, Schwendener and Church, among which Schwendener's theory is the most well-known and surely the most important.".

The footnote mentions a conceivable function of phyllotaxis: "1) Although Wiesner believes to see in the preference of the main sequence an adaptation to light environment, but it seems to me the proof is not given in his work.".

Thus, the apparent lack of functionality was an initial motivation for pursuing the second, causal approach, a mechanical explanation of phyllotaxis. However, the first, evolutionary approach was recently revisited with a suggestion that the arrangement with the golden angle is the most functional when it comes to be rearranged in ranked patterns complying with Schimper-Braun's rule [26] (for an animated demonstration see [27]). At any rate, the presented results are rather compatible with a conventional view that the divergence angle is an inherited characteristic with which natural selection has to do. Evolutionary perspectives should not be readily dismissed on superficial grounds.

Last but not least, although the present study was devoted exclusively to the key problem of the most typical case, qualitative diversity in phyllotaxis should not be disregarded [1-7]. From a broad perspective, there is also good reason to consider that the golden angle is a necessary consequence of developmental dynamics of the shoot apical meristem. Although phyllotaxis patterns are species-specific, the pattern often changes for no apparent reason. Qualitative diversity of phyllotaxis patterns due to ontogenetic changes are well explained by geometrical considerations in terms of variable-sized primordia [28] and dislocations in regular patterns [3]. The causal approach since Hofmeister, in which evolution plays, if any, only a minor role, can provide a parsimonious explanation for all phyllotaxis phenomena, including the most striking phenomenon of the golden angle in spiral phyllotaxis. 


\section{Acknowledgments}

The author received no financial support for this study.

\section{Competing interests}

No competing interests have been declared.

\section{Supplementary material}

The following supplementary material for this article is available online at http://pbsociety.org.pl/journals/index.php/asbp/rt/suppFiles/ asbp.2015.007/0:

1. Appendix S1: the riddle in terms of the golden section.

\section{References}

1. Braun A. Vergleichende Untersuchung über die Ordnung der Schuppen an den Tannenzapfen als Einleitung zur Untersuchung der Blattstellung. Nov Acta Ac CLC. 1831;15:195-402.

2. Zagórska-Marek B. Phyllotactic patterns and transitions in Abies balsamea. Can J Bot. 1985;63:1844-1854. http://dx.doi.org/10.1139/ b85-259

3. Zagórska-Marek B. Phyllotaxic diversity in Magnolia flowers. Acta Soc Bot Pol. 1994;63:117-137. http://dx.doi.org/10.5586/asbp.1994.017

4. Wiss D, Zagórska-Marek B. Geometric parameters of the apical meristem and the quality of phyllotactic patterns in Magnolia flowers. Acta Soc Bot Pol. 2012;81:203-216. http://dx.doi.org/10.5586/asbp.2012.029

5. Szymanowska-Pułka J. Phyllotactic patterns in capitula of Carlina acaulis L. Acta Soc Bot Pol. 1994;63:229-245. http://dx.doi. org/10.5586/asbp.1994.031

6. Kwiatkowska D. Ontogenetic changes in the shoot primary vasculature of Anagallis arvensis L. Acta Soc Bot Pol. 1995;64:213-222. http:// dx.doi.org/10.5586/asbp.1995.028

7. Gola EM. Phyllotactic pattern formation in early stages of cactus ontogeny. Acta Soc Bot Pol. 2006;75:271-279. http://dx.doi.org/10.5586/ asbp. 2006.032

8. Hirmer M. Zur Kenntnis der Schraubenstellungen im Pflanzenreich. Planta. 1931;14:132-206. http://dx.doi.org/10.1007/BF01916598

9. Bravais L, Bravais A. Essai sur la disposition des feuilles curvisériées, Annales des Sciences Naturelles Botanique 1837;7:42-110.

10. van Iterson G. Mathematische und Mikroskopisch-Anatomische Studien über Blattstellungen. Gustav Fischer, Jena; 1907.

11. Fujita T. Statistische Untersuchungern über den Divergenzwinkel bei den schraubigen Organstellungen. Bot Mag Tokyo. 1939;53:194-199. http://dx.doi.org/10.15281/jplantres1887.53.194

12. Snow R. Problems of phyllotaxis and leaf determination. Endeavour. 1955;14:190-199.

13. Kumazawa M, Kumazawa M. Periodic variations of the divergence angle, internode length and leaf shape, revealed by correlogram analysis. Phytomorphology. 1971;21:376-389.

14. Ryan G, Rouse J, Bursill L. Quantitative analysis of sunflower seed packing. J Theor Biol. 1991;147:303-328. http://dx.doi.org/10.1016/ S0022-5193(05)80490-4

15. Couder Y. Initial transitions, order and disorder in phyllotactic patterns: the ontogeny of Helianthus annuus. A case study. Acta Soc Bot Pol. 1998;67:129-150. http://dx.doi.org/10.5586/asbp.1998.016

16. Okabe T. Systematic variations in divergence angle. J Theor Biol 2012;313:20-41. http://dx.doi.org/10.1016/j.jtbi.2012.08.007

17. Matkowski A, Karwowski R, Zagórska-Marek B. Two algorithms of determining the middle point of the shoot apex by surrounding organ primordia positions and their usage for computer measurements of divergence angles. Acta Soc Bot Pol. 1998;67:151-159. http://dx.doi. org/10.5586/asbp.1998.017

18. Church AH. On the relation of phyllotaxis to mechanical laws. London: Williams \& Norgate; 1904.

19. van Iterson G. New studies on phyllotaxis. Proceedings of Koninklijke Nederlandse Akademie van Wetenschappen. Series C. 1960;63:137-150.

20. Fujita T. Zur Kenntnis der Organstellungen im Pflanzenreich. Jpn J Bot. 1942;12:1-55

21. Roberts DW. The origin of Fibonacci phyllotaxis - an analysis of Adler's contact pressure model and Mitchison's expanding apex model. J Theor Biol 1978;74:217-233. http://dx.doi. org/10.1016/0022-5193(78)90073-5

22. Adler I. A model of contact pressure in phyllotaxis. J Theor Biol. 1974;45:1-79. http://dx.doi.org/10.1016/0022-5193(74)90043-5

23. Mitchison GH. Phyllotaxis and the Fibonacci series. Science. 1977;196:270-275. http://dx.doi.org/10.1126/science.196.4287.270

24. Smith RS, Guyomarc'h S, Mandel T, Reinhardt D, Kuhlemeier C, Prusinkiewicz P. A plausible model of phyllotaxis. Proc Natl Acad Sci USA. 2006;103:1301-1306. http://dx.doi.org/10.1073/pnas.0510457103

25. Jönsson H, Heisler MG, Shapiro BE, Meyerowitz EM, Mjolsness E. An auxin-driven polarized transport model for phyllotaxis. Proc Natl Acad Sci USA. 2006;103:1633-1638. http://dx.doi.org/10.1073/ pnas.0509839103

26. Okabe T. Physical phenomenology of phyllotaxis. J Theor Biol. 2011;280:63-75. http://dx.doi.org/10.1016/j.jtbi.2011.03.037

27. Okabe T. Phyllotaxis of plant shoots [Internet]. 2014 [cited 2014 Mar 12]; Available from: http://demonstrations.wolfram.com/ PhyllotaxisOfPlantShoots

28. Zagórska-Marek B, Szpak M. Virtual phyllotaxis and real plant model cases. Funct Plant Biol. 2008;35:1025-1033. http://dx.doi. org/10.1071/FP08076 